\title{
Layer Count Mapping of Multilayer Hexagonal Boron Nitride Thin Films
}

\author{
Nicholas Cross ${ }^{1}$, Ali Mohsin², Lei Liu², Gong Gu², Gerd Duscher ${ }^{1,3}$
}

${ }^{1 .}$ Materials Science and Engineering Department, University of Tennessee, Knoxville, Tennessee 37996, United States

2. Electrical Engineering and Computer Science Department, University of Tennessee, Knoxville, Tennessee 37996, United States

3. Materials Science and Technology Division, Oak Ridge National Laboratory, Oak Ridge, Tennessee 37831, United States

Accurately mapping the layer count of two-dimensional (2D) layer material thin films over large areas (on the scale of $\mathrm{a} \mathrm{m}^{2}$ ) at high lateral spatial resolution can provide valuable insight into material growth mechanisms and material synthesis feedback. Other characterization techniques (i.e. layer fringe counting, atomic force microscopy, and Raman spectroscopy), although all able to provide varying degrees of useful information, each fall short on some of these characterization metrics. ${ }^{1,2}$ Here we detail two complementary techniques using scanning transmission electron microscopy (STEM) to quantify the layer count of hexagonal boron nitride (h-BN) thin films grown by atmospheric-pressure chemical vapor deposition (CVD). The first method relies on annular dark field (ADF), Z-contrast imaging in which, for the thickness regimes of the samples in question, intensity can be directly correlated to number of h-BN layers. ${ }^{3}$ The second method uses electron energy loss spectroscopy (EELS) spectrum mapping and analyzes the boron ionization K-edge to quantify the areal density of boron atoms, which is simple to convert to absolute layer count since the material has a known composition and structure. An advantage of these techniques is that data can be acquired simultaneously and so correlating spatial positions of the sample is not an issue. To give additional confidence, a comparison is performed to determine the amount of discrepancy between these two techniques. The methods detailed here do not require specialized equipment and the TEM available to nearly all materials researchers should be sufficient for replicating these experiments.

Figure 1a provides a qualitative picture of the h-BN films as grown on the copper substrate by SEM imaging. It lacks any quantitative information of the absolute thickness of the h-BN film but what is does show is that there appears to be two growth modes to the h-BN. The first being of a feature with a welldefined triangular shape, indicative of h-BN single crystal growth, and the other being a less uniform growth pattern where the feather are characterized by a high density of non-homogenous patches/clusters. Figure $1 \mathrm{~b}$ and $1 \mathrm{c}$ and layer thickness maps generated by Z-contrast imaging and EELS, respectively. What those two figures show is that there is a continuous monolayer that forms and then secondary adlayers begin to nucleate and grow by the two modes already mentioned. These adlayers seen in figure 1a are one atomic layer and in places they overlap to form small regions of greater than two layers thick. Figure 1d is a map generated to find the discrepancy between figures $1 \mathrm{~b}$ and $1 \mathrm{c}$ and is accomplished by plotting the difference in pixel values between the two (figure 1c is re-binned to match the image pixel dimensions of 1b). We find that the same features seen in figure 1a and replicated in the quantitative thickness maps of figures $1 \mathrm{~b}$ and $1 \mathrm{c}$ and that the two techniques agree very well in the regions that are not obstructed by surface contamination, figure $1 \mathrm{~d}$. 
References:

[1] Li Song, Lijie Ci, and Hao Lu, Nano Letters 10 (2010), p. 3209

[2] Ariel Ismach, Harry Chou, and Domingo Ferrer, ACS Nano 6 (2012), p. 6378

[3] D. E. Jesson and S. J. Pennycook, Proceedings: Mathematical and Physical Sciences 449 (1995), p. 273

[4] The authors acknowledge funding from the NSF grant \#DMR-1410940

(a)

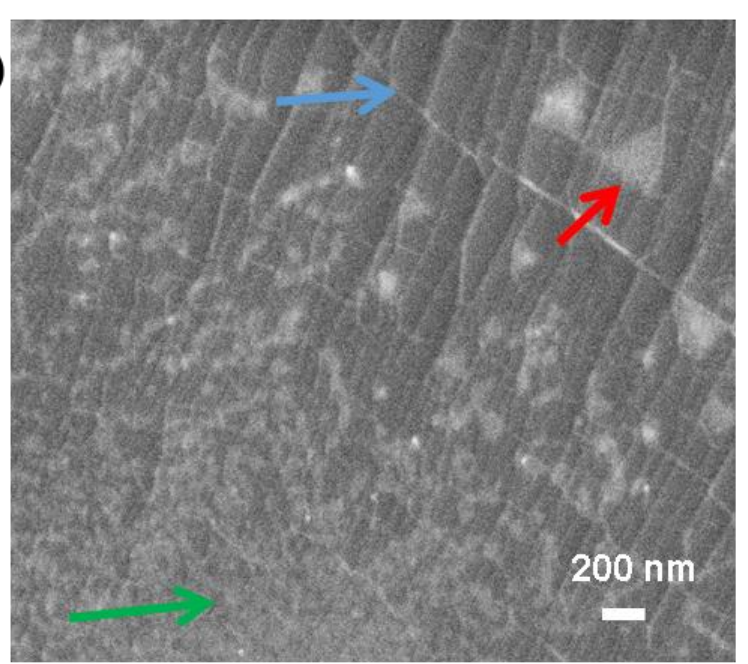

(c)

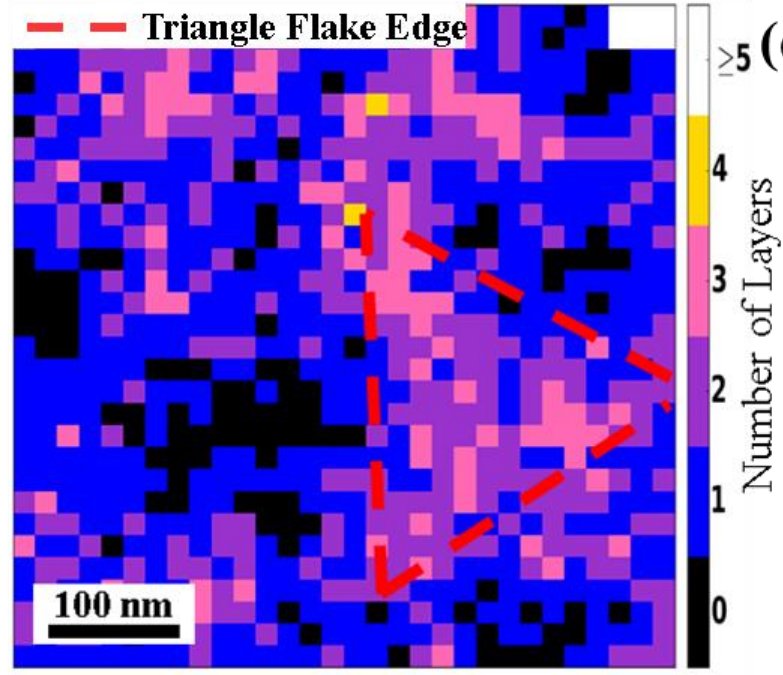

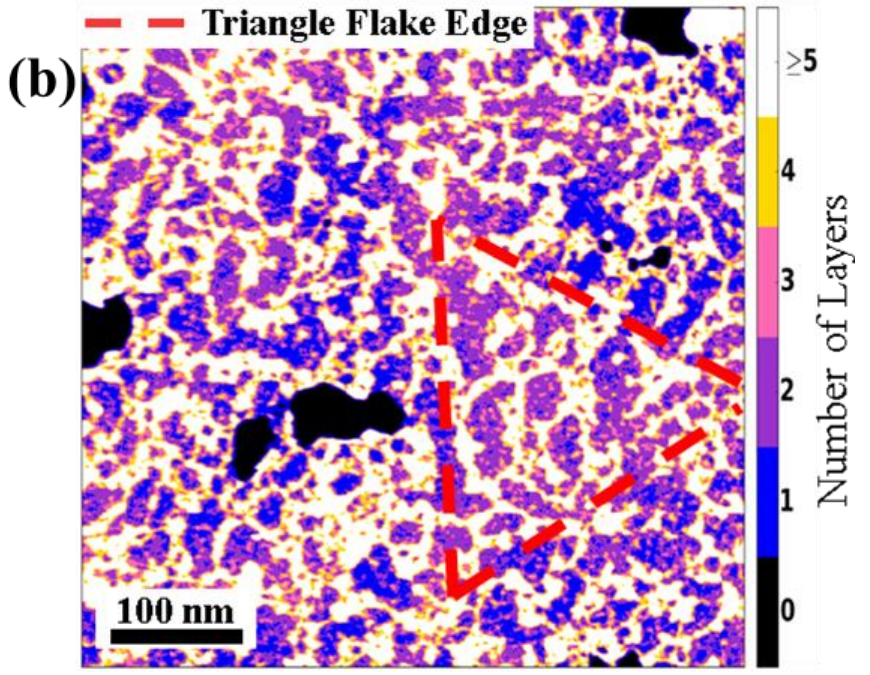

(d)

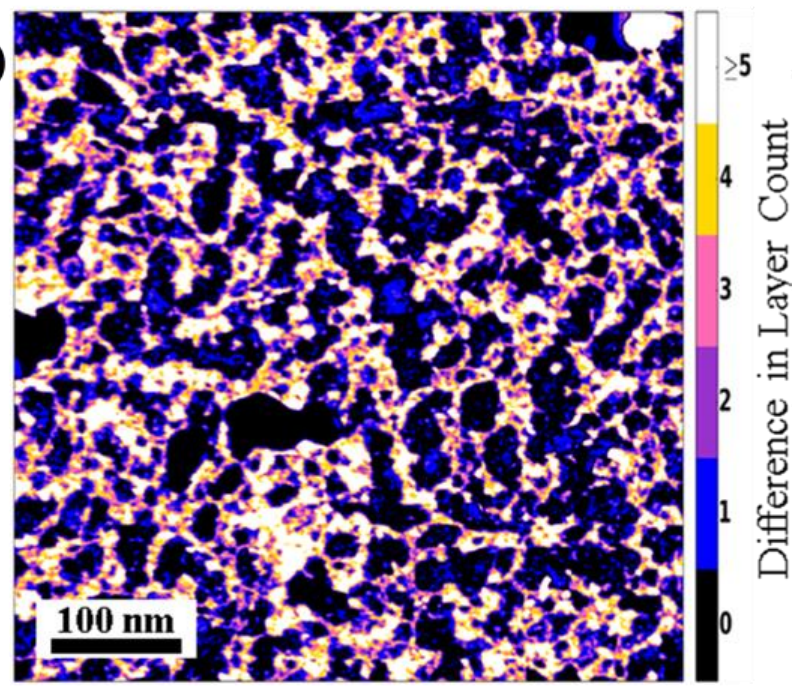

Figure 1. (a) SEM image showing wrinkles in the h-BN film (blue arrows), very dense clusters of adlayers, having sizes of few tens of nm (green arrows), and somewhat isolated triangles with dimensions of few hundreds of nm (red arrows). (b) Layer thick map derived from data attained by Zcontrast imaging. Red dotted contour shows a triangular adlayer having dimensions of roughly $200 \mathrm{~nm}$. Areas where surface contamination is higher, are saturated as white and assigned layer number greater than 5. Black regions represent holes in the film. (c) Layer thickness map derived from data attained by EELS spectrum imaging. Again, a triangular adlayer has been highlighted using a dashed red line. (d) A spatial map that represents the difference between Figures $1 \mathrm{~b}$ and 1c. The regions which are atomically clean agree well which is shown by pixels colored black and areas with contamination have relatively large amounts of discrepancy. 